\title{
Statistical reappraisal of the clinical significance of nail beading in rheumatoid arthritis
}

\author{
E N GRANT, ${ }^{1}$ N BELLAMY, ${ }^{12}$ W W BUCHANAN ${ }^{2}$ E M GRACE, ${ }^{2}$ AND \\ S O'LEAR Y'
}

From the ${ }^{1}$ University of Western Ontario, London, Ontario, Canada; and the ${ }^{2}$ McMaster University Medical Centre, Hamilton, Ontario, Canada

SUMMARY Nail beading has previously been reported as an accompaniment of rheumatoid arthritis. In order to assess the clinical significance of this form of nychodystrophy the fingernails and toenails of 119 patients with rheumatoid arthritis and an equal number of control subjects were studied. Analysis of data based on 4642 nails indicates that the presence of a global pattern of beading (i.e. $\geqslant 50 \%$ involvement of the nail area) on the surface of at least six fingernails or four toenails is highly suggestive of underlying rheumatoid disease. The positive predictive value of these configurations is in the order of $95 \%$. Nail beading, however, is infrequent in early disease and therefore its diagnostic value is limited. Although there is a strong association between nail beading and rheumatoid arthritis, the aetiology and prognostic implications of this clinical sign remain obscure.

Dystrophic nails are a feature of a number of rheumatic diseases and may provide important clues to diagnosis. ${ }^{1}$ Although the typical nychodystrophy of psoriasis is familiar to all practising clinicians, the nail abnormalities which accompany rheumatoid arthritis have received relatively little attention. It was more than 20 years ago that Dr E B D Hamilton reported in this journal the results of a detailed descriptive study of the appearance and growth characteristics of fingernails in patients with rheumatoid arthritis. ${ }^{2} \mathrm{He}$ observed severe beading in two or more fingernails in $27 \%$ of rheumatoid arthritis patients and in $17 \%$ of controls. Although the aetiology and pathogenesis were not defined, it has been postulated that beading may arise as the result of nail bed vasculitis. In order better to define the clinical significance of rheumatoid nychodystrophy we compared the fingernails and toenails of 119 patients with rheumatoid arthritis with those of an equal number of non-rheumatoid controls. The objective of the study was to assess the significance of the proported association with respect to $(a)$ individual nail involvement, $(b)$ multiple nail involvement, $(c)$ the pattern of beading (i.e., focal

Accepted for publication 21 March 1985.

Correspondence to $\operatorname{Dr} \mathrm{N}$ Bellamy. Division of Rheumatology. Victoria Hospital. 777 Baseline Road East, London. Ontario. N6A 4S2, Canada. versus global), and $(d)$ the severity of involvement (none, mild, moderate, or severe).

\section{Patients and methods}

One hundred and nineteen consecutive patients with classic or definite rheumatoid arthritis ${ }^{3}$ and 119 age and sex matched controls with a variety of other musculoskeletal conditions were included in the evaluation. Patients were derived from inpatient and outpatient practices at the two participating centres. The following demographic and disease data were recorded for each patient: age, sex, disease duration, number of American Rheumatism Association (ARA) criteria, and rheumatoid factor status. Details of current and previous drug therapy were obtained by direct questioning, by prompted recall with a pill identification board, ${ }^{4}$ and by a review of hospital charts. Thereafter each fingernail and toenail was separately examined by one of three observers (EG, NB, EG), who looked for the presence of longitudinal beading on the nail surface. Each nail was categorised as follows: $(a)$ no beading, $(b)$ focal beading (i.e., $<50 \%$ of the nail surface involved), or (c) global beading (i.e. $\geqslant 50 \%$ of the nail surface involved). When longitudinal beading was observed, it was graded as either mild, moderate, or severe according to a set of three photographic reference standards. Moderate global nail 


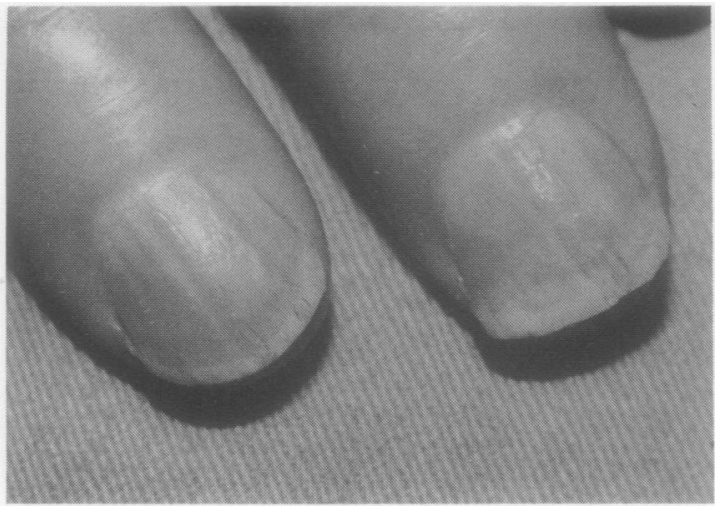

Fig. 1 Moderate global nail beading in a patient with rheumatoid arthritis.

beading in a patient with rheumatoid arthritis is illustrated in Fig. 1. A distinction was made between this type of nychodystrophy and any other types of nail dystrophy observed. Analysis of data was performed as follows. The sensitivity and specificity of longitudinal nail beading were determined by standard statistical techniques ${ }^{5}$ for each grade (none, mild, moderate, and severe), pattern (global only, focal only, and combined focal and global beading), and nail (finger and toe). Further values were calculated to assess the importance of multiple nail involvement. Likelihood ratios ${ }^{6}$ were calculated for many different configurations of nail involvement in order to identify the combination of variables which best discriminated between rheumatoid arthritis patients and controls. In order to correlate $^{7}$ varying degrees of nychodystrophy with age, disease duration, rheumatoid factor status, and duration of specific drug therapies, a nail score was derived for each patient. The nail score was based on the number of fingernails (range 0-10) showing a global pattern of involvement irrespective of its grade.

\section{Results}

The demographic and disease profiles of the study patients are illustrated in Table 1 . Subjects were closely matched by age and sex and can be regarded as equivalent in respect of these demographic variables. The range of disease duration for the rheumatoid population was broad, the mean duration being 13.7 years. Ninety-two of the subjects were seropositive for IgM rheumatoid factor. Of the 119 controls 82 had a variety of degenerative conditions of the peripheral and axial skeleton, eight had connective tissue disorders (CTD), 18 had non-rheumatoid inflammatory arthropathies (sero-
Table 1 Demographic and disease profiles

\begin{tabular}{|c|c|c|}
\hline & $\begin{array}{l}\text { Rheumatoid } \\
\text { arthritis patients }\end{array}$ & $\begin{array}{l}\text { Musculoskeletal } \\
\text { controls }\end{array}$ \\
\hline $\begin{array}{l}\text { No } \\
\text { M/F } \\
\text { Age-mean (range) } \\
\text { Disease duration } \\
\quad \text { (years) } \\
\text { Disease subsets }\end{array}$ & $\begin{array}{l}119 \\
27 / 92 \\
56 \cdot 82 \text { (29-78) } \\
\\
13 \cdot 7 \text { (1 month- } \\
\quad 44 \text { years) } \\
\text { Seropositive } 92 \\
\text { Seronegative } 27\end{array}$ & $\begin{array}{l}\text { Degenerative disease } \\
\text { Connective tissue } \\
\text { disease } 8 \\
\text { Inflammatory } \\
\quad \text { arthropathy } 18 \\
\text { Soft tissue } \\
\text { rheumatism } 11\end{array}$ \\
\hline
\end{tabular}

negative spondylarthritis, crystal synovitis), and $1 \Phi$ had soft tissue rheumatism.

The initial analysis was a digit by digit compariso $\$$ of rheumatoid patients versus controls. Both fingernails (Fig. 2) and toenails (Fig. 3) were compared of the basis of varying degrees of global beading Although global beading was detected in both groups of patients, two important differences were. noted. Firstly, beading was considerably more feso quent in rheumatoid subjects than in controls, secondly, severe focal beading was an exclusfige feature of patients with rheumatoid arthritis. Withio each of the two groups, however, the relativo frequency of involvement of individual nails in the hands and feet was similar, though the thumb nails and to a lesser extent the great toe nails tended to bet involved with slightly greater frequency. Althoug $\mathrm{B}$ no beading was recorded in the left fourth or righp fourth and fifth toe nails in control subjects, should be noted that in some instances it was

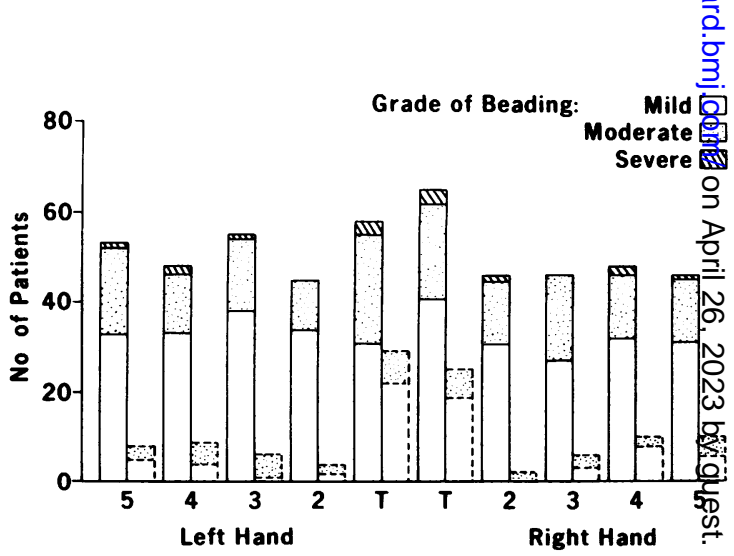

Fig. 2 Digit by digit comparison of global nail beading in fingernails of rheumatoid arthritis patients (solid lines) versus controls (broken lines). 


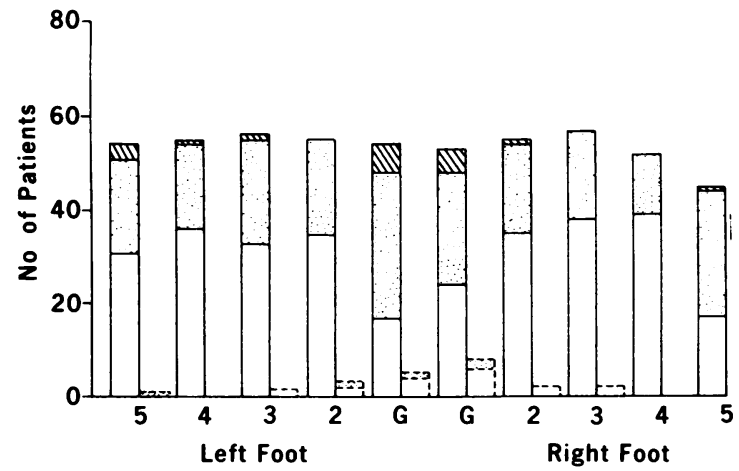

Fig. 3 Digit by digit comparison of global nail beading in toenails of rheumatoid arthritis patients (solid lines) versus controls (broken lines). See Fig. 2 for shading code.

difficult to assess the fourth and fifth toe nails because of their small surface area and undulating surface. The relative frequency with which each grade of nail dystrophy occurred displayed an inverse relationship to the severity of involvement. Thus mild beading was more frequent than moderate beading, which in turn was more frequent than severe beading. Although highly specific for rheumatoid arthritis, the relative infrequency of the severe grade of beading limited the applicability of this clinical sign in differentiating between the two groups of subjects. A similar descriptive analysis was conducted for focal patterns of beading; this was observed less frequently than global beading but with similar frequency in the two study groups.

In the next stage of the analysis our data were compared with those reported by Hamilton ${ }^{2}$ (Table 2). Since Hamilton did not discriminate between moderate and severe degrees of involvement, these two categories were combined and the sensitivity, specificity, likelihood ratio, and positive predictive values calculated based on the involvement of two or more fingernails. Although the sensitivities were similar, our estimate of the specificity was higher
(92\% versus $83 \%)$ and consequently the likelihood ratio was also greater ( 3.5 versus $1 \cdot 6)$. The positive predictive value for our data was $60 \%$ at a disease prevalence of $30 \%$ and $21 \%$ at a disease prevalence of $7 \%$. These two values for disease prevalence were selected from published reports of the prevalence of rheumatoid arthritis in rheumatology consulting practices. $^{89}$ When mild degrees of global beading were also considered in the analysis sensitivity increased to $63 \%$, specificity fell to $76 \%$, and the likelihood ratio declined to 2.6. Since Hamilton's original report did not contain data about toenail beading, a similar comparative analysis against our own data set was not possible.

Sensitivity and specificity may be usefully combined into a single value termed the likelihood ratio. If the likelihood ratio is increased it has the effect of increasing the positive predictive value of a clinical sign. Therefore since it had already been recognised that nail beading involved multiple nails, it was reasoned that accounting for the number of nails involved might favourably influence the positive predictive value of nail beading in identifying patients with rheumatoid arthritis. Likelihood ratios based on a progressively increasing number of toenails or fingernails were calculated and are illustrated in Fig. 4. The likelihood ratio was at a maximum for fingernails when six or more digits were involved. Although the likelihood ratio for toenails increased above six or more nails, the difficulty in assessing the fourth and fifth toes effectively reduced the number of digits which could be easily and reliably assessed to six. For this reason we selected four or more toenails as the preferred level at which the likelihood ratio for lower limb nychodystrophy was at a maximum. The test characteristics for global beading (any grade) involving six or more fingernails (Table 2) were as follows: sensitivity $40 \%$, specificity $98 \%$, likelihood ratio 20 , positive predictive value $89 \%$ at a prevalence of $30 \%$ and $60 \%$ at a prevalence of $7 \%$. In contrast, global beading (any grade) involving four

Table 2 Comparison of sensitivity (Sens), specificity (Spec), likelihood ratio (LR), and positive predictive value $(P P V)$ for different configurations of nail involvement in two reported series

\begin{tabular}{|c|c|c|c|c|c|c|c|}
\hline & \multirow[t]{2}{*}{ Grade* $^{*}$} & \multirow[t]{2}{*}{ No of nails } & \multirow[t]{2}{*}{ Sens $(\%)$} & \multirow[t]{2}{*}{ Spec(\%) } & \multirow[t]{2}{*}{$L R$} & \multicolumn{2}{|c|}{$\begin{array}{l}\text { PPV at prevalence } \\
\text { rates of }\end{array}$} \\
\hline & & & & & & $7 \%$ & $30 \%$ \\
\hline Hamilton $(1960)^{2}$ & Severe & 2 fingernails & 27 & 83 & $1 \cdot 6$ & 11 & 41 \\
\hline Grant (1985) & 2.3 & 2 fingernails & 28 & 92 & $3 \cdot 5$ & 21 & 60 \\
\hline Grant (1985) & 1.2 .3 & 2 fingernails & 63 & 76 & $2 \cdot 6$ & 17 & 53 \\
\hline Grant (1985) & $1,2.3$ & 4 toenails & 51 & 99 & $51 \cdot 0$ & 79 & 95 \\
\hline
\end{tabular}

$\cdot 1=$ mild; 2 =moderate; $3=$ severe. 


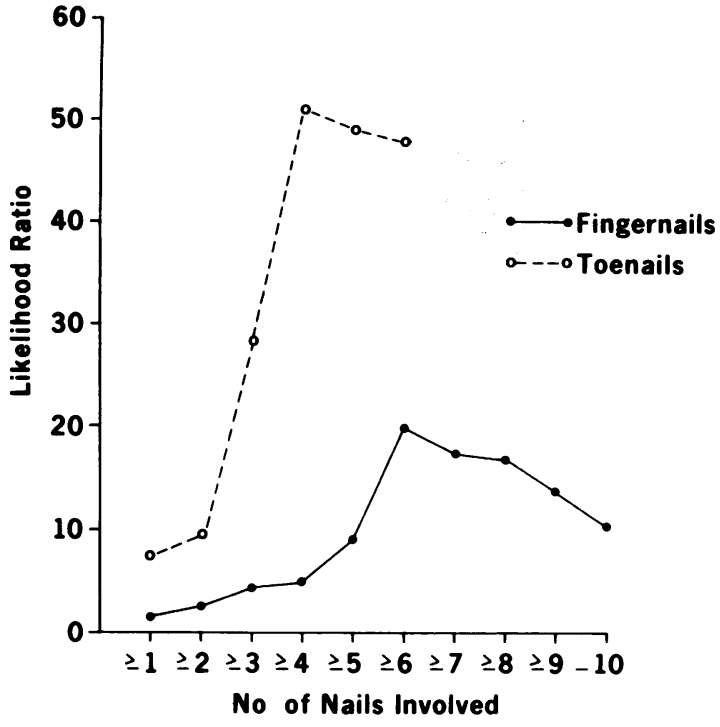

Fig. 4 Likelihood ratios for progressive numbers of nails involved.

or more toenails (Table 2) produced the following characteristics: sensitivity $51 \%$, specificity $99 \%$, likelihood ratio 51 , positive predictive value $95 \%$ at a prevalence of $30 \%$ and $79 \%$ at a prevalence of $7 \%$.

In order to assess the clinical significance of nail beading an aggregate nail score (range $0-10$ ) was derived for each rheumatoid patient by assigning a score of 1 for each finger showing any global pattern of beading and a score of zero for each nail devoid of any such beading. These nail scores were correlated with the patient's age, disease duration, duration of second-line therapy, and rheumatoid factor titre (Table 3). Correlation coefficients varied from $0 \cdot 08$ to $0 \cdot 27$, indicating a general lack of any significant correlation between any disease or demographic variable and the nail score.

Table 3 Correlations of nail score* (fingers) with selected demographic and disease variables

\begin{tabular}{ll}
\hline Variable & Correlation coefficient \\
\hline Age & $0 \cdot 21$ \\
Disease duration & $0 \cdot 27$ \\
Duration of second-line drug therapy & $0 \cdot 11$ \\
Rheumatoid factor titre & 0.08 \\
\hline
\end{tabular}

"Nail score is determined individually by summing total number of nails showing global beading of any grade (i.e., 0 to 10 possible score).

\section{Discussion}

A total of 4642 finger and toenails were examined in 238 patients. The remaining 118 nails were eithe severely deformed and therefore unassessable of had been removed surgically or in some instances lost due to trauma. Although the subdivision of na involvement into focal and global categories was somewhat arbitrary, this decision was vindicated bo the subsequent observation that focal beading occurred with similar frequency in both rheumatoi@ patients and controls. Furthermore since such bead ing is probably due to localised nail bed trauma, it is not surprising that it had little value in differen tiating one group of musculoskeletal patients from the other.

The results of this study confirm the origina $\vec{\Phi}$ observation made by Hamilton ${ }^{2}$ of an association between longitudinal nail beading and rheumatoid arthritis. It is of interest that the calculated indexes for specificity, and particularly for sensitivity, ar $\vec{f}$ similar to those which he reported. We have extended his original observation by noting that the्ठ toenails are similarly affected. Furthermore, the thumb nails and to a lesser extent the great toe nails are more frequently affected than other nails in theifor respective extremities. Although the specificity of this clinical sign was greatest for the toenails, anatomic area proved more difficult to assess. II particular, control subjects frequently had trans verse ridging on the nail surface, which it was felo might obscure any underlying beading. This non specific dystrophy was less of a problem in rheuma $\overrightarrow{\overrightarrow{0}}$ toid patients. The reason for this difference is unclear but it may be speculated that rheumatoid patients employ a more cautious and protective gait may be more restricted in their physical function, op possibly tend to wear modified shoe wear an\& therefore experience less nail trauma than age an sex equivalent individuals with other musculoskelis etal conditions. Since a relatively small increase in the number of control subjects with nail beading would markedly alter the specificity, likelihood ratio, and positive predictive value, we consider that toenail assessments are less reliable and have therefore based our analysis largely on globaw beading in fingernails. By taking account of the tendency of this form of nychodystrophy to involve multiple nails we have been able to optimise the likelihood ratio and thus enhance the positive predictive value of this clinical sign. As previousl noted these positive predictive values have beer adjusted for a disease prevalence ranging from 7 to $30 \%$, since we wished to evaluate the use of naif beading as a clinical sign in physical diagnosis. Thu it was essential to use patients with non-rheumatoif 
musculoskeletal diseases as controls and to adjust the prevalence of rheumatoid arthritis statistically in order to estimate the performance characteristics of this clinical sign when applied in rheumatology outpatient practice. Under these conditions the positive predictive value of global beading in six or more nails ranged from 60 to $89 \%$ and in four or more toenails ranged from 79 to $95 \%$. Unfortunately, however, a high positive predictive value does not necessarily imply high diagnostic value. Thus during the first year of rheumatoid disease, when this physical sign might be of diagnostic value, only $20 \%$ of patients display any evidence of nail beading. In contrast, nail beading tends to occur in the later stages of disease when the diagnosis has already been confirmed. In order to examine some of the potential determinants of nail beading we have correlated the nail score against a number of disease and demographic variables. The lack of any strong correlation between beading and either age, disease duration, duration of second-line therapy, or rheumatoid factor titre suggests that the pathogenesis of this physical sign is dependent on some alternative, albeit, basic feature of the disease process. It has been suggested that beading may simply be age related, ${ }^{2}$ but the poor correlation between age and nail score and the substantial between-group differences noted cannot be accounted for by this hypothesis. Furthermore, although beading is infrequent in the early months of rheumatoid disease, it does not bear a simple relationship to either disease duration or rheumatoid factor positivity. It has previously been speculated that this nychodystrophy may be the result of microvascular disease in the nail bed. Although we can neither confirm nor refute this suggestion, it is of note that no clinical evidence of nail bed vasculitis or systemic vasculitis was detected in any of the rheumatoid patients studied. To date neither the natural history nor the prognostic value of nail beading have been defined but they are the subject of continuing study.

\section{References}

1 Bellamy N, Rooney P J, Buchanan W W. Nail abnormalities in rheumatic disease. Hosp Update 1983; 1: 1997-2001.

2 Hamilton E B D. Nail studies in rheumatoid arthritis. Ann Rheum Dis 1960; 19: 167-73.

3 A Committee of the American Rheumatism Association: revision of diagnostic criteria for rheumatoid arthritis. Arthritis Rheum 1959; 2: 16-20.

4 Bellamy N, Grace E, Hanna B, Grant E, Tugwell P, Buchanan $\mathrm{W} W$. Clinical evaluation of two strategies for improving patient recall of prior drug therapy. Br J Clin Pharmacol 1984; 17: 769-72.

5 Armitage P. Diagnostic tests. In: Statistical methods in medical research. London: Blackwell Scientific, 1980: 433-8.

6 Sackett D L. Clinical epidemiology rounds: interpretation of diagnostic data: How to do it with simple matter. Can Med Assoc J 1983; 129: 947-54.

7 Colton T. Regression and correlation. In: Statistics in medicine. Boston: Little, Brown, 1974: 184-217.

8 Bohan A. The private practice of rheumatology: the first 1000 patients. Arthritis Rheum 1981; 24: 1304-7..

9 Hazanec D J. First year of a rheumatologist in private practice. Arthritis Rheum 1982; 25: 718-9.

\section{Book review}

Clinics in Rheumatic Diseases. The Hand. Vol. 10. No. 3. Edited by C B Wynn Parry. Pp. 746. £15·00. Saunders: Londön. 1984.

Many rheumatologists are frightened of becoming too interested in their patients' hands. Although detailed assessment and a carefully planned course of treatment with surgery at its heart may eventually be rewarding for both physician and patient, such a course of action is time consuming, involves much help from the therapists, and is not always accompanied by useful functional gain and patient satisfaction. The physician's fears are not helped by the confusion that abounds concerning the pathogenic mechanisms responsible for arthritic deformity, the best surgical approaches, the postoperative regimens, and the likely results.

The editor of this volume, one of a series now in its tenth year, has acknowledged all these problems and has deliberately added to the confusion. He has asked a number of international authorities on hand surgery in arthritis to describe different aspects of the treatment, principally in rheumatoid arthritis, but has accepted considerable duplication of material, has not sought agreement about mechanisms, for instance in swan-neck deformity, and has not demanded a simple, uniform postoperative programme.

It is a difficult book to read, partly because of the repetition and partly because much of it seems familiar, so the new is missed. Although rheumatoid arthritis is extensively covered, the interest in osteoarthritis is largely confined to the wrist, and other connective tissue diseases that can produce very difficult hand problems, particularly scleroderma, are almost ignored. The volume is completed by chapters on the overall impact of rheumatic diseases on patients, on a wider consideration of treatment including drugs, and on assessment of hand function, splint making, and joint protection.

Although it has much to commend it, I think it will leave. most rheumatologists frightened. I also think that reading alone will prove to be insufficient and that we shall still require to go with our therapists to see how the experts tackle these problems. At least we now know where to go.

A G Mowat 